\title{
Narrow escape and leakage of Brownian particles
}

\author{
A. Singer $*$ \\ Department of Mathematics and PACM, Princeton University, \\ Fine Hall, Washington Road, Princeton NJ 08544-1000 USA \\ Z. Schust \\ Department of Mathematics, Tel-Aviv University, Tel-Aviv 69978, Israel \\ D. Holcman \\ Department of Mathematics, Weizmann Institute of Science, Rehovot 76100, Israel and \\ Visiting address: Département de Mathématiques et de Biologie, \\ Ecole Normale Supérieure, 46 rue d'Ulm 75005 Paris, France.
}

(Dated: October 31, 2018)

\begin{abstract}
Questions of flux regulation in biological cells raise renewed interest in the narrow escape problem. The often inadequate expansions of the narrow escape time are due to a not so well known fact that the boundary singularity of Green's function for Poisson's equation with Neumann and mixed Dirichlet-Neumann boundary conditions in three-dimensions contains a logarithmic singularity. Using this fact, we find the second term in the expansion of the narrow escape time and in the expansion of the principal eigenvalue of the Laplace equation with mixed Dirichlet-Neumann boundary conditions, with small Dirichlet and large Neumann parts. We also find the leakage flux of Brownian particles that diffuse from a source to an absorbing target on a reflecting boundary of a domain, if a small perforation is made in the reflecting boundary.

PACS numbers: 05.40.-Jc., 87.10.-e
\end{abstract}

\footnotetext{
*Electronic address: amits@math.princeton.edu

$\dagger$ Electronic address: schuss@post.tau.ac.il

${ }^{\ddagger}$ Electronic address: david.holcman@weizmann.ac.il
} 


\section{INTRODUCTION}

The narrow escape problem in diffusion theory, which goes back to Lord Rayleigh [1], is to calculate the mean first passage time, also called the narrow escape time (NET), of a Brownian particle to a small absorbing window on the otherwise reflecting boundary of a bounded domain. The renewed interest in the small hole problem is due to its relevance in molecular biology and biophysics. The small hole often represents a small target on a cellular membrane, such as a protein channel, which is a target for ions [2], a receptor for neurotransmitter molecules in a neuronal synapse [3], a narrow neck in the neuronal spine, which is a target for calcium ions [4], and so on. The physiological role of the small hole is often to regulate flux, which carries a physiological signal. For example, the NMDA channels in the post synaptic membrane in the neuronal cleft are small targets for diffusing glutamate molecules released from a vesicle at the pre synaptic membrane. The leakage problem here is to find the fraction of the released molecules that reach the channels before being irreversibly absorbed by the surrounding medium (e.g., glia transporters) [5], [6] (see also http://en.wikipedia.org/wiki/Chemical_synapse). The position and the number of the NMDA and AMPA receptors regulate synaptic transmission and is believed to be a part of coding memory [3], [7].

The narrow escape problem is connected to that of calculating the principal eigenvalue of the mixed Dirichlet-Neumann problem for the Laplace equation in a bounded domain, whose Dirichlet boundary is only a small patch on the otherwise Neumann boundary. Specifically, the principal eigenvalue is asymptotically the reciprocal of the narrow escape time in the limit of shrinking patch.

The recent history of the problem begins with the work of Ward, Keller, Henshaw, Van De Velde, Kolokolnikov, and Titworth [8, 9, 10, 11] on the principal eigenvalue and is based on boundary layer theory and matched asymptotics, in which the boundary layer equation is the classical electrified disk problem, solved explicitly by Weber in 1873 [12, 13]. The work of Holcman, Singer, Schuss, and Eisenberg [14, 15, 16, 17, 18, 19, 20, 21] on the NET for diffusion with and without a force field and for several small windows and its applications in biology, is based on the known structure of the singularity of Neumann's 
function at the boundary [13, 22, 23, 24] and on the Helmholtz integral equation [25] (see [26]). The most recent work of Bénichou and Voituriez [27] on the NET in diffusion and anomalous diffusion finds the dependence of the NET on the initial point inside the boundary layer and finds the scaling laws for sub-diffusions. In these papers the leading term in the asymptotic expansion was calculated in the shrinking window limit.

Neither the second term, nor its order of magnitude were calculated for the three dimensional problem, except in the case of a spherical domain with a small circular absorbing window, where an explicit solution was constructed by a generalization of Collins' method (an error in the coefficient of the second term, given in [15], is corrected here). The difficulty in finding, or even estimating, the second term can be attributed to the practically unknown (to mathematicians and physicists) structure of the singularity of Neumann's function on the boundary. While classical texts in partial differential equations and in classical mathematical physics [13, 22, 23, 24] mention only the leading order singularity of the Newtonian potential and a regular correction, [23] shows (in an exercise) that Neumann's function for a sphere has a logarithmic singularity at the boundary. The logarithmic boundary singularity of Neumann's function for the Laplace equation in a general regular domain seems to have been discovered by Popov [28] and elaborated by Silbergleit, Mandel, and Nemenman [29] (which cites neither [23] nor [28]).

Another small window problem is that of a leaky conductor of Brownian particles, which is a bounded domain with a source of particles on the boundary or in the interior, and a (big) target, which is an absorbing part of the boundary. The remaining boundary is reflecting. If the boundary has a small absorbing patch (a hole), some of the Brownian particles may leak out and never make it to the big absorbing target. The calculation of the leakage flux is not the same as that in the narrow escape problem, because the total flux on the boundary remains bounded as the small hole shrinks. The calculation of the leakage flux was attempted in [30] for diffusion in a flat cylinder with a source at the reflecting top and a small absorbing window at the reflecting bottom, and absorbing lateral envelop. The three-dimensional diffusion in the cylinder was assumed to be well approximated by radial diffusion in a circular disk. 
In this paper, we find the structure of the boundary singularity of the Neumann function for the Poisson equation and of the Green-Neumann function for the mixed problem (with Dirichlet and Neumann boundary conditions) in a general bounded domain $\Omega$, whose boundary $\partial \Omega$ is sufficiently smooth. Our calculations use the method of [28, 29]. We find that for $\boldsymbol{z} \in \partial \Omega, \boldsymbol{y} \in \Omega \cup \partial \Omega$, the structure of the Neumann function (in dimensionless variables) is

$$
N(\boldsymbol{y}, \boldsymbol{z})=\frac{1}{2 \pi|\boldsymbol{y}-\boldsymbol{z}|}-\frac{1}{8 \pi}[L(\boldsymbol{z})+N(\boldsymbol{z})] \ln |\boldsymbol{y}-\boldsymbol{z}|+v_{S}(\boldsymbol{y}, \boldsymbol{z}),
$$

where $L(\boldsymbol{z})$ and $N(\boldsymbol{z})$ are the principal curvatures of $\partial \Omega$ at $\boldsymbol{z}$ and $v_{S}(\boldsymbol{y}, \boldsymbol{z})$ is a bounded function of $\boldsymbol{x}, \boldsymbol{y}$ in $\Omega$. If $\Omega$ is a ball of radius $R$, the above mentioned result of Kellog [23] is recovered, because $L(\boldsymbol{z})=N(\boldsymbol{z})=\frac{1}{R}$.

We find that the NET through a circular disk of (dimensionless) radius $a$, centered at $\mathbf{0}$ on the boundary, is

$$
E \tau=\frac{|\Omega|}{4 a D\left[1+\frac{L(\mathbf{0})+N(\mathbf{0})}{2 \pi} a \log a+o(a \log a)\right]},
$$

where $D$ is the diffusion coefficient. If $\Omega$ is a ball of radius $R$, then

$$
E \tau=\frac{|\Omega|}{4 a D}\left[1+\frac{a}{\pi R} \log \frac{R}{a}+o\left(\frac{a}{R} \log \frac{R}{a}\right)\right] .
$$

The result (3) corrects that given in [15]. The case of an elliptic window is handled in a straightforward manner, as in [15].

The principal eigenvalue $\lambda_{1}(a) \sim \frac{1}{E \tau}$ of the Laplace equation in $\Omega$ with Dirichlet conditions given on a circular disk of dimensionless radius $a$ and Neumann boundary conditions elsewhere has the asymptotic expansion

$$
\lambda_{1}(a)=\frac{4 a D}{|\Omega|}\left[1+\frac{L(\mathbf{0})+N(\mathbf{0})}{2 \pi} a \log a+o(a \log a)\right] \quad \text { for } \quad a \rightarrow 0 .
$$

The result (4) provides the missing second term and estimate of the remainder, which was not given in $[8,9,10,11,15]$.

For a leaky conductor, we find that the leakage flux through a circular hole of small (dimensionless) radius $\varepsilon$, centered at $\mathbf{0}$, is

$$
J_{\varepsilon}=4 \varepsilon D u_{0}(\mathbf{0})(1+O(\varepsilon \log \varepsilon)),
$$


where $u_{0}(\mathbf{0})$ is the solution of the reduced problem (without the leak) at the hole.

Equation (5) can be viewed as a generalization of (44) in the sense that the factor $|\Omega|^{-1}$ in (41) can be interpreted as the uniform concentration of the Brownian particle in $\Omega$. The uniform concentration is the solution of the stationary diffusion equation problem with Neumann conditions on the entire boundary, which is the reduced problem for narrow escape. Thus the concentration $u_{0}(\boldsymbol{x})$ is a generalization of the fixed concentration $|\Omega|^{-1}$ in (41).

\section{THE SINGULARITY OF NEUMANN'S FUNCTION}

Consider a bounded domain $\Omega \subset \mathbb{R}^{3}$, given by $\Omega=\left\{\boldsymbol{x} \in \mathbb{R}^{3}: F(\boldsymbol{x})<0\right\}$, where $F \in C^{3}\left(\mathbb{R}^{3}\right)$. Our purpose is to determine the singularity of Green's function for the Laplace equation in $\Omega$ with Neumann boundary conditions (called Neumann's function) and of Green's function for the mixed Dirichlet and Neumann boundary conditions.

The Neumann function $N(\boldsymbol{x}, \boldsymbol{y})$ for this domain is the solution of the boundary value problem

$$
\begin{aligned}
\Delta_{\boldsymbol{x}} N(\boldsymbol{x}, \boldsymbol{y}) & =-\delta(\boldsymbol{x}-\boldsymbol{y})+\frac{1}{|\Omega|}, \quad \text { for } \quad \boldsymbol{x}, \boldsymbol{y} \in \Omega \\
\frac{\partial N(\boldsymbol{x}, \boldsymbol{y})}{\partial \nu_{\boldsymbol{x}}} & =0, \quad \text { for } \quad \boldsymbol{x} \in \partial \Omega, \boldsymbol{y} \in \Omega,
\end{aligned}
$$

where $\boldsymbol{\nu}(\boldsymbol{x})$ is the outer unit normal to the boundary $\partial \Omega$. If $\boldsymbol{x}$ or $\boldsymbol{y}$ (or both) are in $\partial \Omega$, then only a half of any sufficiently small ball about a boundary point is contained in $\Omega$, which means that the singularity of Neumann's function is $\frac{1}{2 \pi|\boldsymbol{x}-\boldsymbol{y}|}$. Therefore Neumann's function for $\boldsymbol{y} \in \partial \Omega$ is written as

$$
N(\boldsymbol{x}, \boldsymbol{y})=\frac{1}{2 \pi|\boldsymbol{x}-\boldsymbol{y}|}+v(\boldsymbol{x}, \boldsymbol{y})
$$

where $v(\boldsymbol{x}, \boldsymbol{y})$ satisfies

$$
\Delta \boldsymbol{x} v(\boldsymbol{x}, \boldsymbol{y})=\frac{1}{|\Omega|} \quad \text { for } \quad \boldsymbol{x} \in \Omega, \boldsymbol{y} \in \partial \Omega
$$


and the boundary condition

$$
\frac{\partial v(\boldsymbol{x}, \boldsymbol{y})}{\partial \nu \boldsymbol{x}}=\frac{1}{2 \pi} \frac{\boldsymbol{\nu}(\boldsymbol{x}) \cdot(\boldsymbol{x}-\boldsymbol{y})}{|\boldsymbol{x}-\boldsymbol{y}|^{3}} \quad \text { for } \quad \boldsymbol{x}, \boldsymbol{y} \in \partial \Omega
$$

Green's identity requires the evaluation of two integrals. The first is the volume integral, which by (6) is

$$
\begin{aligned}
\int_{\Omega}\left[N(\boldsymbol{x}, \boldsymbol{y}) \Delta_{\boldsymbol{x}} v(\boldsymbol{x}, \boldsymbol{z})-v(\boldsymbol{x}, \boldsymbol{z}) \Delta_{\boldsymbol{x}} N(\boldsymbol{x}, \boldsymbol{y})\right] d \boldsymbol{x}= & \int_{\Omega} N(\boldsymbol{x}, \boldsymbol{y}) \frac{1}{|\Omega|} d \boldsymbol{x}+v(\boldsymbol{y}, \boldsymbol{z}) \\
& -\frac{1}{|\Omega|} \int_{\Omega} v(\boldsymbol{x}, \boldsymbol{z}) d \boldsymbol{x},
\end{aligned}
$$

and the second is the surface integral, which by (7) is

$$
\begin{aligned}
& \oint_{\partial \Omega}\left[N(\boldsymbol{x}, \boldsymbol{y}) \frac{\partial v(\boldsymbol{x}, \boldsymbol{z})}{\partial \nu \boldsymbol{x}}-v(\boldsymbol{x}, \boldsymbol{z}) \frac{\partial N(\boldsymbol{x}, \boldsymbol{y})}{\partial \nu \boldsymbol{x}}\right] d S_{\boldsymbol{x}}= \\
& \oint_{\partial \Omega}\left[\frac{1}{2 \pi|\boldsymbol{x}-\boldsymbol{y}|}+v(\boldsymbol{x}, \boldsymbol{y})\right] \frac{\boldsymbol{\nu}(\boldsymbol{x}) \cdot(\boldsymbol{x}-\boldsymbol{z})}{2 \pi|\boldsymbol{x}-\boldsymbol{z}|^{3}} d S \boldsymbol{x} .
\end{aligned}
$$

Thus, for $\boldsymbol{z} \in \partial \Omega$ Green's identity gives

$$
\begin{aligned}
v(\boldsymbol{y}, \boldsymbol{z})= & -\frac{1}{|\Omega|} \int_{\Omega}[N(\boldsymbol{x}, \boldsymbol{y})-v(\boldsymbol{x}, \boldsymbol{z})] d \boldsymbol{x} \\
& +\oint_{\partial \Omega}\left[\frac{1}{2 \pi|\boldsymbol{x}-\boldsymbol{y}|}+v(\boldsymbol{x}, \boldsymbol{y})\right] \frac{\boldsymbol{\nu}(\boldsymbol{x}) \cdot(\boldsymbol{x}-\boldsymbol{z})}{2 \pi|\boldsymbol{x}-\boldsymbol{z}|^{3}} d S_{\boldsymbol{x}}
\end{aligned}
$$

To determine the singularity of this integral when $\boldsymbol{y}$ approaches $\boldsymbol{z}$, we use the method of successive approximations to expand $v(\boldsymbol{x}, \boldsymbol{y})$ as

$$
v(\boldsymbol{x}, \boldsymbol{y}) \sim v_{0}(\boldsymbol{x}, \boldsymbol{y})+v_{1}(\boldsymbol{x}, \boldsymbol{y})+v_{2}(\boldsymbol{x}, \boldsymbol{y})+\ldots,
$$

where $v_{i+1}(\boldsymbol{x}, \boldsymbol{y})$ is more regular than $v_{i}(\boldsymbol{x}, \boldsymbol{y})$ (see [29]). For $\boldsymbol{y}$ or $\boldsymbol{z}$ (or both) in $\partial \Omega$, the first term is the most singular part

$$
v_{0}(\boldsymbol{y}, \boldsymbol{z})=\frac{1}{4 \pi^{2}} \oint_{\partial \Omega} \frac{\boldsymbol{\nu}(\boldsymbol{x}) \cdot(\boldsymbol{x}-\boldsymbol{z})}{|\boldsymbol{x}-\boldsymbol{y} \| \boldsymbol{x}-\boldsymbol{z}|^{3}} d S_{\boldsymbol{x}}
$$

To extract its dominant part, we reproduce here, for completeness, the analysis of [28] with only minor modifications. We consider $z \in \partial \Omega$ and assume that the boundary near 
$\boldsymbol{z}$ is sufficiently smooth. Moving the origin to $\boldsymbol{z}$, we set $\boldsymbol{z}=\mathbf{0}$. Taking a sufficiently small patch $\partial \Omega_{\boldsymbol{z}}$ about $\boldsymbol{z}$, we assume that it can be projected orthogonally onto a circular disk $D_{a}$ of radius $a$ in the tangent plane to $\partial \Omega$ at $\boldsymbol{z}$. We can assume, therefore, that $\partial \Omega_{\boldsymbol{z}}$ can be represented as

$$
x_{3}=f_{\boldsymbol{z}}\left(x_{1}, x_{2}\right)=\frac{1}{2} L(\boldsymbol{z}) x_{1}^{2}+\frac{1}{2} N(\boldsymbol{z}) x_{2}^{2}+o\left(x_{1}^{2}+x_{2}^{2}\right) \quad \text { for } \quad\left(x_{1}, x_{2}\right) \in D_{a} .
$$

If $a$ is sufficiently small, then $o\left(x_{1}^{2}+x_{2}^{2}\right) \ll L x_{1}^{2}+N x_{2}^{2}$. This canonical representation (14) assumes that $\partial \Omega_{\boldsymbol{z}}$ has at least one non-zero curvature and that the quadratic part in Taylor's expansion of $f\left(x_{1}, x_{2}\right)$ about the origin is represented in principal axes.

The asymptotically dominant part as $\boldsymbol{y} \rightarrow \boldsymbol{z}$ is determined by the integral over the patch $\partial \Omega_{\boldsymbol{z}}$, which we write as

$$
v_{0}(\boldsymbol{y}, \mathbf{0}) \sim \frac{1}{4 \pi^{2}} \int_{\partial \Omega_{\boldsymbol{z}}} \frac{\boldsymbol{\nu}(\boldsymbol{x}) \cdot \boldsymbol{x} d S_{\boldsymbol{x}}}{\sqrt{\left(x_{1}-y_{1}\right)^{2}+\left(x_{2}-y_{2}\right)^{2}+\left(x_{3}-y_{3}\right)^{2}}\left[x_{1}^{2}+x_{2}^{2}+x_{3}^{2}\right]^{3 / 2}}
$$

In the representation (14)

$$
\begin{aligned}
\boldsymbol{\nu}(\boldsymbol{x}) & =\frac{\left(L(\boldsymbol{z}) x_{1}, N(\boldsymbol{z}) x_{2},-1\right)+o\left(\sqrt{x_{1}^{2}+x_{2}^{2}}\right)}{\sqrt{1+L^{2}(\boldsymbol{z}) x_{1}^{2}+N^{2}(\boldsymbol{z}) x_{2}^{2}}} \\
\boldsymbol{\nu}(\boldsymbol{x}) \cdot \boldsymbol{x} & =\frac{L(\boldsymbol{z}) x_{1}^{2}+N(\boldsymbol{z}) x_{2}^{2}-x_{3}}{\sqrt{1+L^{2}(\boldsymbol{z}) x_{1}^{2}+N^{2}(\boldsymbol{z}) x_{2}^{2}}} \\
d S_{\boldsymbol{x}} & =\sqrt{1+\left|\nabla f_{\boldsymbol{z}}\right|^{2}} d x_{1} d x_{2} \sim \sqrt{1+L^{2}(\boldsymbol{z}) x_{1}^{2}+N^{2}(\boldsymbol{z}) x_{2}^{2}} d x_{1} d x_{2},
\end{aligned}
$$

so that

$$
v_{0}(\boldsymbol{y}, \mathbf{0}) \sim \frac{1}{4 \pi^{2}} \int_{D_{a}} \frac{\left(L(\boldsymbol{z}) x_{1}^{2}+N(\boldsymbol{z}) x_{2}^{2}-x_{3}\right) d x_{1} d x_{2}}{\sqrt{\left(x_{1}-y_{1}\right)^{2}+\left(x_{2}-y_{2}\right)^{2}+\left(x_{3}-y_{3}\right)^{2}}\left[x_{1}^{2}+x_{2}^{2}+x_{3}^{2}\right]^{3 / 2}} .
$$

The patch $\partial \Omega_{\boldsymbol{z}}$ is represented in polar coordinates in $D_{a}$ as

$$
\left(x_{1}, x_{2}, x_{3}\right)=\left(r \cos \phi, r \sin \phi, r^{2}\left(\frac{L(\boldsymbol{z})}{2} \cos ^{2} \phi+\frac{N(\boldsymbol{z})}{2} \sin ^{2} \phi+o(1)\right)\right),
$$

so transforming $\boldsymbol{y}$ into spherical coordinates

$$
\left(y_{1}, y_{2}, y_{3}\right)=|\boldsymbol{y}|\left(\sin \theta \cos \phi_{0}, \sin \theta \sin \phi_{0}, \cos \theta\right)
$$


we can write (16) as

$$
v_{0}(\boldsymbol{y}, \mathbf{0}) \sim \frac{1}{4 \pi^{2}} \int_{0}^{2 \pi} I(|\boldsymbol{y}|, \phi, \theta) d \phi
$$

where

$$
\begin{aligned}
& I(|\boldsymbol{y}|, \phi, \theta)=\int_{0}^{a} \frac{\left[\frac{1}{2} L r^{2} \cos ^{2} \phi+\frac{1}{2} N r^{2} \sin ^{2} \phi+o\left(r^{2}\right)\right] r d r}{\left[r^{2}+|\boldsymbol{y}|^{2}-2 r|\boldsymbol{y}| \sin \theta \cos \left(\phi-\phi_{0}\right)+O\left(r^{2}|\boldsymbol{y}|+r^{4}\right)\right]^{1 / 2}\left[r^{2}+O\left(r^{4}\right)\right]^{3 / 2}} \\
\sim & \frac{1}{2}\left[L \cos ^{2} \phi+N \sin ^{2} \phi\right] \int_{0}^{a} \frac{d r}{\left[r^{2}+|\boldsymbol{y}|^{2}-2 r|\boldsymbol{y}| \sin \theta \cos \left(\phi-\phi_{0}\right)\right]^{1 / 2}} .
\end{aligned}
$$

Integration with respect to $r$ gives

$$
\begin{aligned}
& \int_{0}^{a} \frac{d r}{\left[r^{2}+|\boldsymbol{y}|^{2}-2 r|\boldsymbol{y}| \sin \theta \cos \left(\phi-\phi_{0}\right)\right]^{1 / 2}}= \\
& \ln \frac{a-|\boldsymbol{y}| \sin \theta \cos \left(\phi-\phi_{0}\right)+\sqrt{a^{2}+|\boldsymbol{y}|^{2}-2 a|\boldsymbol{y}| \sin \theta \cos \left(\phi-\phi_{0}\right)}}{|\boldsymbol{y}|\left(1-\sin \theta \cos \left(\phi-\phi_{0}\right)\right)}= \\
& \ln \frac{1}{|\boldsymbol{y}|}+O(1),
\end{aligned}
$$

for $\boldsymbol{y} \neq \mathbf{0}$. It follows from (18) that for $\boldsymbol{y} \neq \boldsymbol{z}$ the leading order singularity is

$$
v_{0}(\boldsymbol{y}, \boldsymbol{z}) \sim \frac{1}{8 \pi}(L(\boldsymbol{z})+N(\boldsymbol{z})) \ln \frac{1}{|\boldsymbol{y}-\boldsymbol{z}|}+O(1) .
$$

For further analysis of the $O(1)$ term, see [29].

The canonical representation (14) of a hemisphere of (dimensionless) radius $R$ at the south pole is $x_{3}=R-\sqrt{R^{2}-\left(x_{1}^{2}+x_{2}^{2}\right)}$, so $L(\boldsymbol{z})=N(\boldsymbol{z})=\frac{1}{R}$. Therefore, for $|\boldsymbol{z}|=R$,

$$
N(\boldsymbol{y}, \boldsymbol{z})=\frac{1}{2 \pi|\boldsymbol{y}-\boldsymbol{z}|}+\frac{1}{4 \pi R} \ln \frac{1}{|\boldsymbol{y}-\boldsymbol{z}|}+O(1),
$$

in agreement with [23, p.247, Exercise 4].

\section{APPLICATION TO THE NARROW ESCAPE PROBLEM}

\section{A. Escape through a small circular hole}

As mentioned in the Introduction, the narrow escape problem [8, 9, 10, 11, 14, 15, 16, 17, 18] is to calculate the mean escape time of a Brownian particle from a bounded 
domain $\Omega$, whose boundary is reflecting, except for a small absorbing patch (or patches [19, 20]) $\partial \Omega_{a}$. We assume here that $\partial \Omega_{a}$ is a circular disk of radius $a \ll|\Omega|^{1 / 3}$ and that a ball of radius $R \gg a$ can be rolled on $\partial \Omega$ inside $\Omega$. This means that there are no narrow passages in $\Omega$. We denote $\partial \Omega_{r}=\partial \Omega-\partial \Omega_{a}$ and $\varepsilon=a /|\Omega|^{1 / 3}$ and investigate the limit $\varepsilon \rightarrow 0$. We assume that all coordinates have been scaled with $|\Omega|^{1 / 3}$, so that all variables and parameters are dimensionless.

The MFPT $u(\boldsymbol{x})$ from a point $\boldsymbol{x} \in \Omega$ to $\partial \Omega_{a}$ is the solution of the mixed boundary value problem

$$
\begin{gathered}
\Delta u(\boldsymbol{x})=-\frac{1}{D}, \quad \text { for } \quad \boldsymbol{x} \in \Omega \\
u(\boldsymbol{x})=0 \quad \text { for } \quad \boldsymbol{x} \in \partial \Omega_{a} \\
\frac{\partial u(\boldsymbol{x})}{\partial \nu_{\boldsymbol{x}}}=0 \quad \text { for } \quad \boldsymbol{x} \in \partial \Omega_{r},
\end{gathered}
$$

where $D$ is the diffusion coefficient. The compatibility condition,

$$
\int_{\partial \Omega_{a}} \frac{\partial u(\boldsymbol{x})}{\partial \nu \boldsymbol{x}} d S_{\boldsymbol{x}}=-\frac{|\Omega|}{D}
$$

is obtained by integrating (22) over $\Omega$ and using (23) and (24).

Green's identity and the boundary conditions (77), (23), and (24) give

$$
u(\boldsymbol{y})-\frac{1}{D} \int_{\Omega} N(\boldsymbol{x}, \boldsymbol{y}) d \boldsymbol{x}=\int_{\partial \Omega} N(\boldsymbol{x}, \boldsymbol{y}) \frac{\partial u(\boldsymbol{x})}{\partial \nu} d S_{\boldsymbol{x}}+C,
$$

where

$$
C=\frac{1}{|\Omega|} \int_{\Omega} u(\boldsymbol{x}) d \boldsymbol{x} .
$$

Following the argument in [15], we note that $N(\boldsymbol{x}, \boldsymbol{y})$ is an integrable function independent of $\partial \Omega_{a}$, whose integral is uniformly bounded, whereas $C \rightarrow \infty$ as $\varepsilon \rightarrow 0$. Setting $g(\boldsymbol{x})=$ $\frac{\partial u(\boldsymbol{x})}{\partial \nu_{\boldsymbol{x}}}$ for $\boldsymbol{x} \in \partial \Omega_{a}$ and using the boundary condition (23), we obtain from (26) the integral equation for the flux density $g(\boldsymbol{x})$ in $\partial \Omega_{a}$,

$$
\int_{\partial \Omega_{a}} N(\boldsymbol{x}, \boldsymbol{y}) g(\boldsymbol{x}) d S_{\boldsymbol{x}}=-C \quad \text { for } \quad \boldsymbol{y} \in \partial \Omega_{a}
$$


which, in view of (8) , (20) now becomes the generalized Helmholtz equation [25], [15]

$$
\begin{aligned}
& \int_{\partial \Omega_{a}} g(\boldsymbol{x})\left[\frac{1}{2 \pi|\boldsymbol{x}-\boldsymbol{y}|}+H(\boldsymbol{x}, \boldsymbol{y}) \log |\boldsymbol{x}-\boldsymbol{y}|+O(1)\right] d S_{\boldsymbol{x}}=-C \text { for } \boldsymbol{y} \in \partial \Omega_{a}, \\
& H(\boldsymbol{x}, \boldsymbol{y})=-\frac{1}{8 \pi}[L(\boldsymbol{y})+N(\boldsymbol{y})] \sim-\frac{1}{8 \pi}[L(\mathbf{0})+N(\mathbf{0})], \quad \text { for } \quad \boldsymbol{x}, \boldsymbol{y} \in \partial \Omega_{a} \quad \text { for } \quad \varepsilon \rightarrow 0,
\end{aligned}
$$

where $L(\mathbf{0}), N(\mathbf{0})$ are the principal curvatures at the center $\mathbf{0}$ of $\partial \Omega_{a}$. To solve (29), we expand $g(\boldsymbol{x})=g_{0}(\boldsymbol{x})+g_{1}(\boldsymbol{x})+g_{2}(\boldsymbol{x})+\cdots$, where $g_{i+1}(\boldsymbol{x}) \ll g_{i}(\boldsymbol{x})$ for $\varepsilon \rightarrow 0$ and choose

$$
g_{0}(\boldsymbol{x})=\frac{-2 C}{a \pi \sqrt{1-\frac{|\boldsymbol{x}|^{2}}{a^{2}}}}
$$

It was shown in [1], [26], [15] that if $\partial \Omega_{a}$ is a circular disk of radius $a$, then

$$
\frac{1}{2 \pi} \int_{\partial \Omega_{a}} \frac{g_{0}(\boldsymbol{x})}{|\boldsymbol{x}-\boldsymbol{y}|} d S_{\boldsymbol{x}}=C \quad \text { for all } \boldsymbol{y} \in \partial \Omega_{a}
$$

It follows that $g_{1}(\boldsymbol{x})$ satisfies the integral equation

$$
\frac{1}{2 \pi} \int_{\partial \Omega_{a}} \frac{g_{1}(\boldsymbol{x})}{|\boldsymbol{x}-\boldsymbol{y}|} d S \boldsymbol{x}=\frac{2 C}{a \pi} \int_{\partial \Omega_{a}} \frac{H(\boldsymbol{x}, \boldsymbol{y}) \log |\boldsymbol{x}-\boldsymbol{y}|}{\sqrt{1-\frac{|\boldsymbol{x}|^{2}}{a^{2}}}} d S \boldsymbol{x}
$$

Setting $y=a \boldsymbol{\eta}, \boldsymbol{x}=a \boldsymbol{\xi}$, and changing to polar coordinates in the integral on the right hand side of (32), we obtain

$$
\frac{1}{2 \pi} \int_{\partial \Omega_{a}} \frac{g_{1}(\boldsymbol{x})}{|\boldsymbol{x}-\boldsymbol{y}|} d S_{\boldsymbol{x}}=\frac{2 C a^{2}}{a \pi} \int_{0}^{2 \pi} d \theta \int_{0}^{1} \frac{H(a \boldsymbol{\xi}, a \boldsymbol{\eta})[\log a+\log |\boldsymbol{\xi}-\boldsymbol{\eta}|]}{\sqrt{1-r^{2}}} r d r
$$

which gives in the limit $\varepsilon \rightarrow 0$ (e.g., keeping $|\Omega|$ fixed and $a \rightarrow 0$ ) that

$$
\frac{1}{2 \pi} \int_{\partial \Omega_{a}} \frac{g_{1}(\boldsymbol{x})}{|\boldsymbol{x}-\boldsymbol{y}|} d S_{\boldsymbol{x}}=-\frac{C[L(\mathbf{0})+N(\mathbf{0})]}{2 \pi} a \log a+o(a \log a) .
$$

As in the pair (30), (31), we obtain that

$$
g_{1}(\boldsymbol{x})=\frac{-C[L(\mathbf{0})+N(\mathbf{0})]}{\pi^{2} \sqrt{1-\frac{|\boldsymbol{x}|^{2}}{a^{2}}}} \log a+o(\log a) .
$$


Finally, to determine the asymptotic value of the constant $C$, we recall that $g(\boldsymbol{x})=\frac{\partial u(\boldsymbol{x})}{\partial \nu \boldsymbol{x}}$ and use in (25) the approximation

$$
g(\boldsymbol{x}) \sim g_{0}(\boldsymbol{x})+g_{1}(\boldsymbol{x}) \sim \frac{-2 C}{a \pi \sqrt{1-\frac{|\boldsymbol{x}|^{2}}{a^{2}}}}\left[1+\frac{L(\mathbf{0})+N(\mathbf{0})}{2 \pi} a \log a\right] .
$$

We obtain the narrow escape time $E \tau=C$ (in dimensionless variables) as

$$
E \tau=\frac{|\Omega|}{4 a D\left[1+\frac{L(\mathbf{0})+N(\mathbf{0})}{2 \pi} a \log a+o(a \log a)\right]} .
$$

The principal eigenvalue $\lambda_{1}(a) \sim \frac{1}{E \tau}$ of the Laplace equation in $\Omega$ with the mixed Dirichlet-Neumann boundary conditions (23), (24) has the asymptotic expansion for $\varepsilon \rightarrow 0$

$$
\lambda_{1}(a)=\frac{4 a D}{|\Omega|}\left[1+\frac{L(\mathbf{0})+N(\mathbf{0})}{2 \pi} a \log a+o(a \log a)\right] .
$$

The result (38) provides the missing second term and estimate of the remainder, which was not given in $[8,9,10,11,15]$.

If $\Omega$ is a ball of radius $R$, then $L(\mathbf{0})+N(\mathbf{0})=\frac{2}{R}$ and the narrow escape time $E \tau=C$ is given (in dimensional variables) by

$$
E \tau=\frac{|\Omega|}{4 a D\left[1-\frac{a}{\pi R} \log \frac{R}{a}+o\left(\frac{a}{R} \log \frac{R}{a}\right)\right]}=\frac{|\Omega|}{4 a D}\left[1+\frac{a}{\pi R} \log \frac{R}{a}+o\left(\frac{a}{R} \log \frac{R}{a}\right)\right]
$$

The result (39) corrects that given in [15]. Specifically, equation (3.52) in [15] is missing the factor $1 / \pi$ of equation (39), which should have been carried from eq.(3.51) in [15]. The case of an elliptic window is handled in a straightforward manner, as in [15].

\section{B. Leakage in a conductor of Brownian particles}

A conductor of Brownian particles is a bounded domain $\Omega$, with a source of particles on the boundary or in the interior and a target, which is an absorbing part $\partial \Omega_{a}$ of $\partial \Omega$. The remaining boundary $\partial \Omega_{r}$ is reflecting. Some of the Brownian particles may leak out of $\Omega$ if $\partial \Omega_{r}$ contains a small absorbing hole $S(\varepsilon)$. The calculation of the leakage flux is not 
the same as that in the narrow escape problem, because the total flux on the boundary remains bounded as the small hole shrinks. Our purpose is to find the portion that leaks through the small hole out of the total flux.

The (dimensionless) stationary density $u(\boldsymbol{x})$ of the Brownian particles satisfies the mixed boundary value problem

$$
\begin{aligned}
D \Delta u(\boldsymbol{x}) & =0 \quad \text { for } \quad \boldsymbol{x} \in \Omega \\
\left.\frac{\partial u(\boldsymbol{x})}{\partial \nu}\right|_{\partial \Omega_{r}} & =0 \\
-\left.D \frac{\partial u(\boldsymbol{x})}{\partial \nu}\right|_{\partial \Omega_{s}} & =\phi(\boldsymbol{x}) \\
\left.u(\boldsymbol{x})\right|_{\partial \Omega_{a}} & =\left.u(\boldsymbol{x})\right|_{S(\varepsilon)}=0,
\end{aligned}
$$

where $\phi(\boldsymbol{x})$ is the flux density of the source on the boundary. Next, we derive an asymptotic expression for the flux through $S(\varepsilon)$,

$$
J_{\varepsilon}=D \int_{S(\varepsilon)} \frac{\partial u(\boldsymbol{x})}{\partial \nu} d S \boldsymbol{x},
$$

in terms of the solution $u_{0}(\boldsymbol{x})$ of the reduced problem (without $S(\varepsilon)$ ), thus avoiding the need to construct boundary layers. First, we find the flux of each eigenfunction and then, using eigenfunction expansion, we calculate $J_{\varepsilon}$. Every eigenfunction $u_{\varepsilon}(\boldsymbol{x})$ of the homogeneous problem (40) satisfies

$$
\begin{aligned}
-D \Delta u_{\varepsilon}(\boldsymbol{x}) & =\lambda(\varepsilon) u_{\varepsilon}(\boldsymbol{x}) \text { for } \quad \boldsymbol{x} \in \Omega \\
\frac{\partial u_{\varepsilon}(\boldsymbol{x})}{\partial \nu} & =0 \quad \text { for } \quad \boldsymbol{x} \in \partial \Omega_{s} \cup \partial \Omega_{r} \\
u_{\varepsilon}(\boldsymbol{x}) & =0 \quad \text { for } \quad \boldsymbol{x} \in S(\varepsilon) \cup \partial \Omega_{a} .
\end{aligned}
$$

The matched asymptotics method of [8]-[11] gives the expansion of the eigenvalues

$$
\lambda(\varepsilon)=\lambda(0)+\lambda_{1} \varepsilon+o(\varepsilon),
$$


where $\lambda(0)$ is the eigenvalue of the reduced problem (for $\Omega$ without any small holes).

We define the reduced Green function (without the small hole) as the solution of the mixed boundary value problem with $D=1$,

$$
\begin{aligned}
-\Delta G(\boldsymbol{x}, \boldsymbol{y}) & =\delta(\boldsymbol{x}-\boldsymbol{y}) \quad \text { for } \quad \boldsymbol{x}, \boldsymbol{y} \in \Omega \\
\frac{\partial G}{\partial \nu}(\boldsymbol{x}, \boldsymbol{y}) & =0 \quad \text { for } \quad \boldsymbol{x} \in \partial \Omega_{s} \cup \Omega_{r}, \boldsymbol{y} \in \Omega \\
G(\boldsymbol{x}, \boldsymbol{y}) & =0, \quad \text { for } \quad \boldsymbol{x} \in \partial \Omega_{a}, \boldsymbol{y} \in \Omega .
\end{aligned}
$$

Multiplying (46) by $u_{\varepsilon}(\boldsymbol{y})$ and integrating over $\Omega$, we get

$$
u_{\varepsilon}(\boldsymbol{x})=\frac{\lambda(\varepsilon)}{D} \int_{\Omega} G(\boldsymbol{x}, \boldsymbol{y}) u_{\varepsilon}(\boldsymbol{y}) d \boldsymbol{y}+\int_{S(\varepsilon)} G(\boldsymbol{x}, \boldsymbol{y}) \frac{\partial u_{\varepsilon}(\boldsymbol{y})}{\partial \nu} d S \boldsymbol{y} .
$$

In view of the boundary condition (44), we get from (49) for all $\boldsymbol{x} \in S(\varepsilon)$

$$
\frac{\lambda(\varepsilon)}{D} \int_{\Omega} G(\boldsymbol{x}, \boldsymbol{y}) u_{\varepsilon}(\boldsymbol{y}) d \boldsymbol{y}=-\int_{S(\varepsilon)} G(\boldsymbol{x}, \boldsymbol{y}) \frac{\partial u_{\varepsilon}(\boldsymbol{y})}{\partial \nu} d S \boldsymbol{y} .
$$

The integral on the left hand side of (50) can be expanded about the center of $S(\varepsilon)$ in the form

$$
\int_{\Omega} \lambda(\varepsilon) G(\boldsymbol{x}, \boldsymbol{y}) u_{\varepsilon}(\boldsymbol{y}) d \boldsymbol{y}=G_{0}(\varepsilon)+O(|\boldsymbol{x}|) \quad \text { for } \quad \boldsymbol{x} \in S(\varepsilon),
$$

where the origin is assumed to be in the center of $S(\varepsilon)$ and the $\left(x_{1}, x_{2}\right)$ plane is that of $S(\varepsilon)$.

As in Section III A, Green's function for the mixed boundary value problem has the form

$$
G(\boldsymbol{x}, \boldsymbol{y})=\frac{1}{2 \pi|\boldsymbol{x}-\boldsymbol{y}|}+H(\boldsymbol{x}, \boldsymbol{y}) \log |\boldsymbol{x}-\boldsymbol{y}|+v_{S}(\boldsymbol{x}, \boldsymbol{y})
$$

for $\boldsymbol{x} \in \partial \Omega, \boldsymbol{y} \in \Omega \cup \partial \Omega$, where $H(\boldsymbol{x}, \boldsymbol{y})$ depends locally on the curvatures of the boundary and $v_{S}(\boldsymbol{x}, \boldsymbol{y})$ is a continuous function of $\boldsymbol{x}, \boldsymbol{y} \in \Omega$ and on $\partial \Omega$. We assume that $H(\boldsymbol{x}, \boldsymbol{y})$ is bounded. Using (52) and the expansion (51) in (50), we obtain the Helmholtz equation

$$
\frac{G_{0}(\varepsilon)}{D}+O(|\boldsymbol{x}|)=-\int_{S(\varepsilon)}\left[\frac{1}{2 \pi|\boldsymbol{x}-\boldsymbol{y}|}+H(\boldsymbol{x}, \boldsymbol{y}) \log |\boldsymbol{x}-\boldsymbol{y}|+v_{S}(\boldsymbol{x}, \boldsymbol{y})\right] \frac{\partial u_{\varepsilon}(\boldsymbol{y})}{\partial \nu} d S \boldsymbol{y} .(53)
$$


The leading order singularity of $G(\boldsymbol{x}, \boldsymbol{y})$ and (31) suggest the expansion

$$
\frac{\partial u_{\varepsilon}(\boldsymbol{y})}{\partial \nu}=\frac{C_{0}(\varepsilon)}{\sqrt{1-\frac{|\boldsymbol{y}|^{2}}{\varepsilon^{2}}}}+O(|\boldsymbol{y}|) \text { for } \boldsymbol{y} \in S(\varepsilon),
$$

where $C_{0}(\varepsilon)$ is yet an undetermined coefficient, that is,

$$
\begin{aligned}
\frac{G_{0}(\varepsilon)}{D}+O(|\boldsymbol{x}|)= & -\int_{S(\varepsilon)}\left[\frac{1}{2 \pi|\boldsymbol{x}-\boldsymbol{y}|}+H(\boldsymbol{x}, \boldsymbol{y}) \log |\boldsymbol{x}-\boldsymbol{y}|+v_{S}(\boldsymbol{x}, \boldsymbol{y})\right] \times \\
& {\left[\frac{C_{0}(\varepsilon)}{\sqrt{1-\frac{|\boldsymbol{y}|^{2}}{\varepsilon^{2}}}}+O(|\boldsymbol{y}|)\right] d S \boldsymbol{y} }
\end{aligned}
$$

which reduces at $\boldsymbol{x}=\mathbf{0}$ to

$$
\begin{aligned}
\frac{G_{0}(\varepsilon)}{D}= & \frac{-C_{0}(\varepsilon) \pi \varepsilon}{2}+\int_{S(\varepsilon)} O(|\boldsymbol{y}|)\left[\frac{1}{2 \pi|\boldsymbol{y}|}+H(0, \boldsymbol{y}) \log |\boldsymbol{y}|\right] d S \boldsymbol{y}- \\
& \int_{S(\varepsilon)} \frac{C_{0}(\varepsilon)\left[H(\mathbf{0}, \boldsymbol{y}) \log |\boldsymbol{y}|+v_{S}(\mathbf{0}, \boldsymbol{y})\right] d S \boldsymbol{y}}{\sqrt{1-\frac{|\boldsymbol{y}|^{2}}{\varepsilon^{2}}}}+\int_{S(\varepsilon)} O(|\boldsymbol{y}|) d S \boldsymbol{y} .
\end{aligned}
$$

It follows that

$$
\frac{G_{0}(\varepsilon)}{D}=-\left(\frac{\pi \varepsilon}{2}+O\left(\varepsilon^{2} \log \varepsilon\right)\right) C_{0}(\varepsilon)+O\left(\varepsilon^{2} \log \varepsilon\right),
$$

so that

$$
C_{0}(\varepsilon)=-\frac{G_{0}(\varepsilon)+O\left(\varepsilon^{2} \log \varepsilon\right)}{D\left[\frac{\pi \varepsilon}{2}+O\left(\varepsilon^{2} \log \varepsilon\right)\right]} .
$$

Now, (54) gives the flux through $S(\varepsilon)$ as

$$
\begin{aligned}
-D \int_{S(\varepsilon)} \frac{\partial u_{\varepsilon}(\boldsymbol{y})}{\partial \nu} d S \boldsymbol{y}= & \frac{G_{0}(\varepsilon)+O\left(\varepsilon^{2} \log \varepsilon\right)}{\frac{\pi \varepsilon}{2}+O\left(\varepsilon^{2} \log \varepsilon\right)} \int_{S(\varepsilon)} \frac{d S \boldsymbol{y}}{\sqrt{1-\frac{|\boldsymbol{y}|^{2}}{\varepsilon^{2}}}}+ \\
& \int_{S(\varepsilon)} O(|\boldsymbol{y}|) d S \boldsymbol{y} \\
= & 4 \varepsilon \frac{G_{0}(\varepsilon)+O\left(\varepsilon^{2} \log \varepsilon\right)}{1+O(\varepsilon \log \varepsilon)}+O\left(\varepsilon^{2} \log \varepsilon\right) .
\end{aligned}
$$


To determine $G_{0}(\varepsilon)$, we integrate (42), to get the total flux condition

$$
\lambda(\varepsilon) \int_{\Omega} u_{\varepsilon}(\boldsymbol{x}) d \boldsymbol{x}=D \int_{S(\varepsilon)} \frac{\partial u_{\varepsilon}(\boldsymbol{y})}{\partial \nu} d S \boldsymbol{y}+D \int_{\partial \Omega_{a}} \frac{\partial u_{\varepsilon}(\boldsymbol{y})}{\partial \nu} d S \boldsymbol{y} .
$$

We also recall that (48) implies that

$$
\int_{\partial \Omega_{a}} G(\boldsymbol{x}, \boldsymbol{y}) \frac{\partial u_{\varepsilon}(\boldsymbol{y})}{\partial \nu} d S \boldsymbol{y}=0 \quad \text { for } \quad \boldsymbol{x} \in \partial \Omega_{a},
$$

hence, using equations (50) and (58), we get the two equations

$$
\begin{gathered}
\lambda(\varepsilon) \int_{\Omega} u_{\varepsilon}(\boldsymbol{x}) d \boldsymbol{x}=4 \varepsilon \frac{G_{0}(\varepsilon)+O\left(\varepsilon^{2} \log \varepsilon\right)}{1+O(\varepsilon \log \varepsilon)}+O\left(\varepsilon^{2} \log \varepsilon\right) \\
+D \int_{\partial \Omega_{a}} \frac{\partial u_{\varepsilon}(\boldsymbol{y})}{\partial \nu} d S \boldsymbol{y} \\
\lambda(\varepsilon) \int_{\Omega} G(\mathbf{0}, \boldsymbol{y}) u_{\varepsilon}(\boldsymbol{y}) d \boldsymbol{y}=G_{0}(\varepsilon) .
\end{gathered}
$$

This gives

$$
\begin{aligned}
& \lambda(\varepsilon) \int_{\Omega} u_{\varepsilon}(\boldsymbol{x}) d \boldsymbol{x}= \\
& \frac{4 \varepsilon \lambda(\varepsilon) \int_{\Omega} G(\mathbf{0}, \boldsymbol{y}) u_{\varepsilon}(\boldsymbol{y}) d \boldsymbol{y}+O\left(\varepsilon^{2} \log \varepsilon\right)}{1+O(\varepsilon \log \varepsilon)}+O\left(\varepsilon^{2} \log \varepsilon\right)+D \int_{\partial \Omega_{a}} \frac{\partial u_{\varepsilon}(\boldsymbol{y})}{\partial \nu} d S \boldsymbol{y} .
\end{aligned}
$$

Solving for $\lambda(\varepsilon)$, we find that

$$
\begin{aligned}
\lambda(\varepsilon)= & \frac{D \int_{\partial \Omega_{a}} \frac{\partial u_{\varepsilon}(\boldsymbol{y})}{\partial \nu} d S_{\boldsymbol{y}}+O\left(\varepsilon^{2} \log \varepsilon\right)}{\int_{\Omega} u_{\varepsilon}(\boldsymbol{x}) d \boldsymbol{x}-\frac{4 \varepsilon}{1+O(\varepsilon \log \varepsilon)} \int_{\Omega} G(\mathbf{0}, \boldsymbol{y}) u_{\varepsilon}(\boldsymbol{y}) d \boldsymbol{y}+O\left(\varepsilon^{2} \log \varepsilon\right)} \\
= & \frac{D \int_{\partial \Omega_{a}} \frac{\partial u_{\varepsilon}(\boldsymbol{y})}{\partial \nu} d S \boldsymbol{y}}{\int_{\Omega} u_{\varepsilon}(\boldsymbol{x}) d \boldsymbol{x}}\left(1+\frac{4 \varepsilon \int_{\Omega} G(\mathbf{0}, \boldsymbol{y}) u_{\varepsilon}(\boldsymbol{y}) d \boldsymbol{y}}{\int_{\Omega} u_{\varepsilon}(\boldsymbol{x}) d \boldsymbol{x}}\right)+O\left(\varepsilon^{2} \log \varepsilon\right) .
\end{aligned}
$$


Note that

$$
\frac{D \int_{\partial \Omega_{a}} \frac{\partial u_{\varepsilon}(\boldsymbol{y})}{\partial \nu} d S \boldsymbol{y}}{\int_{\Omega} u_{\varepsilon}(\boldsymbol{x}) d \boldsymbol{x}}=\lambda(0)+O(\varepsilon),
$$

due to the contribution of the boundary layer near $S(\varepsilon)$.

Obviously, $u_{\varepsilon} \rightarrow u_{0}$ as $\varepsilon \rightarrow 0$, where $u_{0}$ is the corresponding eigenfunction of the reduced problem (in the absence of the small hole, see also [8]), so

$\lim _{\varepsilon \rightarrow 0} \int_{\Omega} G(\boldsymbol{x}, \boldsymbol{y}) u_{\varepsilon}(\boldsymbol{y}) d \boldsymbol{y}=\int_{\Omega} G(\boldsymbol{x}, \boldsymbol{y}) u_{0}(\boldsymbol{y}) d \boldsymbol{y}, \quad \lim _{\varepsilon \rightarrow 0} \int_{\partial \Omega_{a}} \frac{\partial u_{\varepsilon}(\boldsymbol{y})}{\partial \nu} d S \boldsymbol{y}=\int_{\partial \Omega_{a}} \frac{\partial u_{0}(\boldsymbol{y})}{\partial \nu} d S \boldsymbol{y}$. Therefore, using (59)-(61) in (57), we find that the flux of $u_{\varepsilon}(\boldsymbol{x})$ through the small hole is

$$
\begin{aligned}
J(\varepsilon) & =-D \int_{S(\varepsilon)} \frac{\partial u_{\varepsilon}(\boldsymbol{y})}{\partial \nu} d S \boldsymbol{y}=4 \varepsilon \lambda(0) \int_{\Omega} G(\mathbf{0}, \boldsymbol{y}) u_{0}(\boldsymbol{y}) d \boldsymbol{y}+O\left(\varepsilon^{2} \log \varepsilon\right) \\
& =4 \varepsilon D u_{0}(\mathbf{0})+O\left(\varepsilon^{2} \log \varepsilon\right) .
\end{aligned}
$$

Finally, expanding the solution $u(\boldsymbol{x})$ of (40) in eigenfunctions, we obtain from (62)

$$
J_{\varepsilon}=4 \varepsilon D u_{0}(\mathbf{0})(1+O(\varepsilon \log \varepsilon))
$$

where $u_{0}(\boldsymbol{x})$ is the solution of the reduced problem (40). In dimensional variables, we obtain

$$
J_{\varepsilon}=4 a D p_{0}(\mathbf{0})+O\left(\frac{a^{2}}{|\Omega|^{2 / 3}} \log \frac{a}{|\Omega|^{1 / 3}}\right),
$$

where $p_{0}(\mathbf{0})$ is the value of the reduced stationary density (without the perforation) at the hole.

\section{SUMMARY AND DISCUSSION}

The main results of this paper are (i) the explicit calculation of the second term in the expansion of the NET, which can be quite significant, and which also provides a bound for the remainder in the expansion; (ii) an explicit expression for the leakage flux through a 
small opening in the impermeable envelope of a conductor of ions. The leakage is often a key control mechanism of physiological function, such as in the synaptic cleft of a neuron, as mentioned in the Introduction. The leakage formula (64) can give explicit expressions for the flux when the reduced problem is explicitly solvable, e.g., in simple geometries. If there are several leaks, at $\boldsymbol{x}_{i}$, then (64) gives

$$
J_{\varepsilon}=4 a D \sum_{i} p_{0}\left(\boldsymbol{x}_{i}\right)+O\left(\frac{a^{2}}{|\Omega|^{2 / 3}} \log \frac{a}{|\Omega|^{1 / 3}}\right)
$$

which demonstrates the role of clustering or un-clustering of the leaks in regulating flux [19], [20]. Specific applications of the results of this paper to molecular biology and biophysics will be published in a separate paper.

\section{Acknowledgments}

AS thanks the Yale University-Weizmann Institute Joint Research Fund; ZS was partially supported by a research grant from TAU; DH was partially supported by an ERCstarting grant and an HFSP research grant.

[1] J. W. S. Baron Rayleigh, The Theory of Sound, Vol. 2, 2nd Ed., Dover, New York, 1945.

[2] B.Hille, Ionic Channels of Excitable Membranes, 3-rd edtn, Sinauer Associates Inc. Sunderland, MA 2001.

[3] G.M. Elias, R.A. Nicoll, "Synaptic trafficking of glutamate receptors by MAGUK scaffolding proteins", Trends Cell Biol. 17 (7), pp.343-52 (2007).

[4] E. Korkotian,D. Holcman, M. Segal, "Dynamic Regulation of Spine-Dendrite Coupling in Cultured Hippocampal Neurons", Euro J. of Neuroscience, 20 (10), pp.2649-63 (2004).

[5] B.L. Patton, A.Y. Chiu, J.R. Sanes, "Synaptic laminin prevents glial entry into the synaptic cleft", Nature 393, pp.698-701 (1998).

[6] V. Gallo and R. Chittajallu, "Unwrapping Glial Cells from the Synapse: What Lies Inside?", Science 292 (5518), pp.872-873 (2001). 
[7] R. Malinow, "AMPA receptor trafficking and long-term potentiation", Philos. Trans. R. Soc. Lond. B Biol. Sci. 358 (1432), pp.707-714 (2003).

[8] M.J. Ward and J.B. Keller, "Strong Localized Perturbations of Eigenvalue Problems", SIAM J. Appl. Math. 53, pp.770-798 (1993).

[9] M.J. Ward, W.D. Henshaw and J.B. Keller, "Summing Logarithmic Expansions for Singularly Perturbed Eigenvalue Problems", SIAM J. Appl. Math., 53, pp.799-828 (1993).

[10] M.J. Ward and E. Van De Velde, "The Onset of Thermal Runaway in Partially Insulated or Cooled Reactors", IMA J. Appl. Math., 48, pp.53-85 (1992).

[11] T. Kolokolnikov, M. Titcombe and M.J. Ward, "Optimizing the Fundamental Neumann Eigenvalue for the Laplacian in a Domain with Small Traps", European J. Appl. Math. 16, pp.161-200 (2005).

[12] H. Weber, "Über die Besselschen Functionen und ihre Anwendung auf die Theorie der elektrischen Ströme". Journal für die reine und angewandte Mathematik, 75, pp.75-105, (1873).

[13] J.D. Jackson, Classical Electrodynamics, 3-rd Edition, Wiley, NY (1998).

[14] D. Holcman and Z. Schuss, "Escape through a small opening: receptor trafficking in a synaptic membrane", J. of Statistical Physics 117 (5/6), pp.191-230 (2004).

[15] A. Singer Z, Schuss, D. Holcman, B. Eisenberg, "Narrow Escape I", J. Stat. Phys. 122 (3), pp.437-463 (2006).

[16] A. Singer Z, Schuss, D. Holcman, "Narrow Escape II", J. Stat. Phys. 122 (3), pp.465-489 (2006)

[17] A. Singer Z, Schuss, D. Holcman, "Narrow Escape III", 122 (3), pp.491-509, (2006).

[18] Schuss Z, Singer A, Holcman D. "The narrow escape problem for diffusion in cellular microdomains", Proc Natl Acad Sci USA, 104 (41), pp.16098-103 (2007).

[19] D. Holcman and Z. Schuss, "Diffusion through a cluster of small windows and flux regulation in microdomains", Phys. Lett. A 372, pp.3768-3772 (2008).

[20] D. Holcman and Z. Schuss, "Diffusion escape through a cluster of small absorbing windows", J. Phys. A: Math. and Theoretical 41155001 (2008). 
[21] A. Singer and Z. Schuss, "Activation through a narrow opening", Phys. Rev. E (Rapid Comm.) 74, pp.020103(R) (2006).

[22] R. Courant and D. Hilbert, Methods of Mathematical Physics, Wiley-Interscience, NY (1989).

[23] O.D. Kellog, Foundations of Potential Theory, Dover Publications, NY 1954.

[24] P.R. Garabedian, Partial Differential Equations, Wiley, NY 1964.

[25] H. L. F. von Helmholtz, Crelle, Bd. 7 (1860).

[26] A.I. Lur'e, Three-Dimensional Problems of the Theory of Elasticity, Interscience Publishers, NY 1964.

[27] O. Bénichou and R. Voituriez, Narrow-Escape Time Problem: Time Needed for a Particle to Exit a Confining Domain through a Small Window", Phys. Rev. Lett. 100, 168105 (2008).

[28] I.Yu. Popov, "Extension theory and localization of resonances for domains of trap type", Math. USSR Sbornik 71 (1), pp.209-234 (1992).

[29] A. Silbergleit, I. Mandel, I. Nemenman, "Potential and field singularity at a surface point charge", J. Math. Phys. 44 (10), pp.4460-4466 (2003).

[30] L.P. Savtchenko and D.A. Rusakov, "The optimal height of the synaptic cleft", Proc. Nat. Acad. Sci. 104 (6), pp.1823-1828 (2007). 